\title{
Pl@ntNet Services, a Contribution to the Monitoring and Sharing of Information on the World Flora
}

Pierre Bonnet ${ }^{\ddagger}, \S$, Julien Champl, Hervé Goëau ${ }^{\ddagger} \S$, Fabian-Robert Stöterl, Benjamin Deneul,§, Maximilien Servajean", Antoine Affouard ${ }^{, \S}$, Jean-Christophe Lombardo', Oleksandra Levchenko', Hugo Gressel,§, Alexis Jolyl

‡ CIRAD, UMR AMAP, F-34398 Montpellier, France

$\S$ AMAP, Univ Montpellier, CIRAD, CNRS, INRAE, IRD, Montpellier, France

| Inria, LIRMM, Montpellier, France

I LIRMM, Univ Montpellier, CNRS, AMIS, Université Paul Valéry, Montpellier, France

Corresponding author: Pierre Bonnet (pierre.bonnet@cirad.fr)

Received: 24 Sep 2020 | Published: 25 Sep 2020

Citation: Bonnet P, Champ J, Goëau H, Stöter F-R, Deneu B, Servajean M, Affouard A, Lombardo J-C,

Levchenko O, Gresse H, Joly A (2020) PI@ntNet Services, a Contribution to the Monitoring and Sharing of Information on the World Flora. Biodiversity Information Science and Standards 4: e58933.

https://doi.org/10.3897/biss.4.58933

\begin{abstract}
Pl@ntNet is a scientific and citizen platform based on artificial intelligence techniques to help participants more easily identify plants with their smartphones. The identification of plant species is indeed an important step for many scientific, educational and land management activities (for natural or cultivated spaces). This step, which is integrated into various biology training courses, is difficult to develop on a large scale, even for professionals. This difficulty in naming plants by a very large part of the society limits the positive interactions between humans and their environment, and thus reduces awareness of the contribution of plants to the well-being of humanity. It is therefore essential to attempt to solve this problem on a large geographical, taxonomic and sociological scale in order to develop more responsible and environmentally precautionary societies.
\end{abstract}


In the framework of the European Cos4Cloud project (2019 - 2023), which involves several European citizen science platforms (such as iSpot in the UK, Natusfera in Spain or Artporta len in Sweden), PI@ntNet is developing innovative digital services aimed at:

1. facilitating the integration of automated species identification into other citizen science portals and

2. enabling researchers to use PI@ntNet data and tools for their own research.

The services implemented will be provided on the European Open Science Cloud (EOSC), to increase the capacity and interest of European scientists to implement citizen science projects aimed at contributing to the sustainable development goals identified by the United Nations.

The tools currently available on the PI@ntNet monitoring platform, as well as the data extraction methodologies that have enabled the publication of two large datasets on the Global Biodiversity Information Facility (GBIF) portal, will be presented. In particular, we will present our latest advances allowing:

- the adaptation of PI@ntNet services to nature reserves or botanical gardens,

- the monitoring of target species for a given geographical area,

- the prediction of species distribution at the national scale, based on high-resolution remote sensing imagery.

The results of the LifeCLEF campaign, an annual international scientific and technological benchmark based on PI@ntNet data, will be presented to compare the latest machinelearning techniques used to enable automated species identification and species prediction based on geolocation. The fruit of this work, involving a large multi-disciplinary team will illustrate the benefits of working at the frontier between biological, digital and citizen sciences.

\section{Keywords}

citizen science, multimedia biodiversity data, plant diversity, plant identification, biodiversity informatics, artificial intelligence.

\section{Presenting author}

Pierre Bonnet

\section{Presented at}

TDWG 2020 


\section{Funding program}

This project has received funding from the European Union's Horizon 2020 research and innovation programme under grant agreement $\mathrm{No}^{\circ} 863463$ (Cos4Cloud project), the support of Agropolis Fondation, and \#DigitAG. 